\title{
Early New Physics with Jets at the LHC
}

\author{
Matthias U. Mozer* \\ on behalf of the CMS and ATLAS Collaborations \\ Frije Universiteit Brussel \\ Pleinlaan 2 \\ 1050 Brussels \\ Belgium \\ E-mail: mmozerdvub.ac.be
}

\begin{abstract}
Many proposed models of new physics predict yet undiscovered particles which decay with high branching fractions into quarks and/or gluons, such as extra gauge bosons or fourth generation quarks. The high branching fractions to quarks make searches in channels with jets in the final state attractive for investigations at low integrated luminosities or new phenomena with low production cross sections. The most challenging aspect of such an analysis is the large background expected from standard model QCD processes. The prospects of new discoveries in final states with jets at the ATLAS and CMS experiments at the LHC will be presented.
\end{abstract}

The 2009 Europhysics Conference on High Energy Physics,

July 16 - 222009

Krakow, Poland

${ }^{*}$ Speaker. 


\section{Introduction}

Various proposed models of new physics include yet undiscovered particles which have decay modes that lead to jets in their final state. While jet final states are likely to suffer from high QCD induced backgrounds, they also typically have higher branching fractions than possible leptonic decays. Particles that decay to jet final states are also likely to interact via the strong nuclear force and can be pair-produced in gluon-gluon fusion, a process that promises high production cross sections at hadron colliders such as the LHC. For these reasons it appears promising to search for new particles at the LHC. The ATLAS [1] and CMS [目] experiments are particularly suitable for such searches as they were designed for the highest possible acceptance and a wide variety of final states.

\section{Expected Limits and Discovery Reach}

The following examples show an overview of possible new physics detectable with the ATLAS and CMS detector during early data taking at the LHC.

\subsection{Generic High Mass Dijet Resonances}

A variety of models predict resonances decaying into two color-charged particles, leading to a two-jet final state. The resonance itself can be a heavy gauge boson [3] or an excited quark [4]. A direct search for such a resonance, however, is plagued by a large background due the large dijet cross section from ordinary QCD processes. A promising method to enhance the sensitivity of a search in view of this large background has been studied by the CMS collaboration [5]. This study exploits the different angular distributions expected from a resonance decay (resulting jets are mostly central, details depending on the resonance spin) and standard model QCD processes (cross section steeply rising in the forward direction). Assuming a beam energy at the LHC of $7 \mathrm{TeV}, 100 \mathrm{pb}^{-1}$ of integrated luminosity give the CMS experiment a discovery reach for excited quarks of $\sim 2 \mathrm{TeV} / c^{2}$.

\subsection{Leptoquarks}

Leptoquarks arise in various Grand Unified Theories, Technicolor and composite models [6] and carry non-zero baryon and lepton numbers simultaneously. They are predicted to occur in three generations, corresponding to the three lepton and quark generations of the standard model. Intergenerational coupling of leptoquarks is highly constrained by the experimental limits on flavorchanging neutral currents and proton decay [6, 7].

At the LHC, leptoquarks are dominantly produced as pairs in gluon-gluon fusion and quark anti-quark annihilation processes. The final state is thus expected to contain two leptons of the same flavor and opposite charge as well as two jets.

The ATLAS and CMS experiments have prepared searches for leptoquarks in these final states [8, 9, 10], concentrating on 1st and 2nd generation leptoquarks. Though these analysis differ in some details, they all proceed with the same strategy: leptons and jets are identified and events matching the expected final state selected. The background is reduced by imposing quality and isolation criteria on the reconstructed jets and leptons as well as the requirement that the 
total transverse energy of the final state must reach a certain threshold. Leptons and jets are then combined to pairs to obtain an invariant mass spectrum of the potential leptoquarks.

The resulting sensitivity of the experiments depends crucially on the branching fraction $\beta$ of the leptoquark into the lepton and quark final state (as opposed to the neutrino quark final state). Assuming a value of $\beta=1$ the ATLAS experiment at $7 \mathrm{TeV}$ beam energy is expected to be sensitive to first and second generation leptoquarks up to $600 \mathrm{GeV} / c^{2}$ mass with an integrated luminosity of $123 \mathrm{pb}^{-1}$ and $103 \mathrm{pb}^{-1}$, respectively. With an integrated luminosity of $100 \mathrm{pb}^{-1}$, discovery of a first generation leptoquark at CMS should be possible up to a leptoquark mass of about 505, 370 and $270 \mathrm{GeV} / c^{2}$ assuming, respectively, $\beta=1,0.5$, and 0.3 . Under the same conditions, discovery of a second generation leptoquark at CMS should be possible up to a leptoquark mass of about 470 and $250 \mathrm{GeV} / c^{2}$ assuming, respectively, $\beta=1$ and 0.3 . Figure 11 shows a more detailed view of the possible discoveries.

\subsection{4th Generation Quarks}

The production of 4 th generation quarks at the LHC is expected to be dominated by gluongluon fusion processes. The $b$-like quark $\left(b^{\prime}\right)$ is expected to be the lighter of the possible 4 th generation quarks and thus more likely to be accessible at the LHC. In searches for such a $b^{\prime}$ quark, it is useful to distinguish between light $b^{\prime}$ quarks (decay $b^{\prime} \rightarrow t W$ kinematically forbidden) and a heavy $b^{\prime}$ (decay $b^{\prime} \rightarrow t W$ allowed). The CMS experiment has prepared searches for both of these scenarios [11, 12].

A light $b^{\prime}$ is expected at tree-level to decay as $b^{\prime} \rightarrow c W$, a decay channel that is experimentally very challenging due to the large expected backgrounds. However, as the CKM element $V_{b^{\prime} c}$ is not known and may be small, it is possible that penguin decays such as $b^{\prime} \rightarrow b Z$ have a sizable branching fraction. The presented search [11] focuses on events where at least one member of the $b^{\prime}$ pair decays through this channel, selecting events that contain at least one candidate for a leptonic $Z$ decay plus a candidate for a leptonic $W$ decay (from the other member of the pair) and at least two jets of transverse momentum above $30 \mathrm{GeV} / c$. This selection effectively removes most backgrounds and it is expected that a light $b^{\prime}$ can be excluded at $95 \%$ confidence level at $5 \mathrm{TeV}$ beam energy using an integrated luminosity of $200 \mathrm{pb}^{-1}$ at a mass below $190 \mathrm{GeV} / c^{2}$ assuming a branching fraction of the $b^{\prime} \rightarrow b Z$ decay vs the $b^{\prime} \rightarrow t W$ decay of $10 \%$ vs $90 \%$

A heavy $b^{\prime}$ is expected to dominantly decay to a top quark and $W$ boson, where the top quarks in turn decay to $b$ quarks and $W$ bosons. The high multiplicity of these final states pose several challenges: The acceptance for the complete final state in the detector is rather low, leading to low event yields even at high production cross sections. Additionally, the final state particles are expected to have a low transverse momentum compared to the mass of the heavy quark, a kinematic domain prone to standard model background. The analysis presented in [12] requires at least two well identified and isolated leptons of the same charge, thus selecting events in which at least two of the four expected $W$ bosons decay leptonically. The di-lepton mass is required to differ from the $\mathrm{Z}$ mass by at least $10 \mathrm{GeV} / c^{2}$ to veto background from charge-misidentification. Additionally, at least 4 jets are required with a transverse momentum above $35 \mathrm{GeV} / c$. A sample of 200(60) $\mathrm{pb}^{-1}$ of integrated luminosity at $5 \mathrm{TeV}$ beam energy is expected to exclude $b^{\prime}$ quarks of a mass up to $485(405) \mathrm{GeV} / c^{2}$ at $95 \%$ confidence level. Additionally the CMS experiment has prepared a search for an exotic $t^{\prime}$ quark of charge $4 / 3$, degenerate in mass with the corresponding $b^{\prime}$ [13, 14]. The 

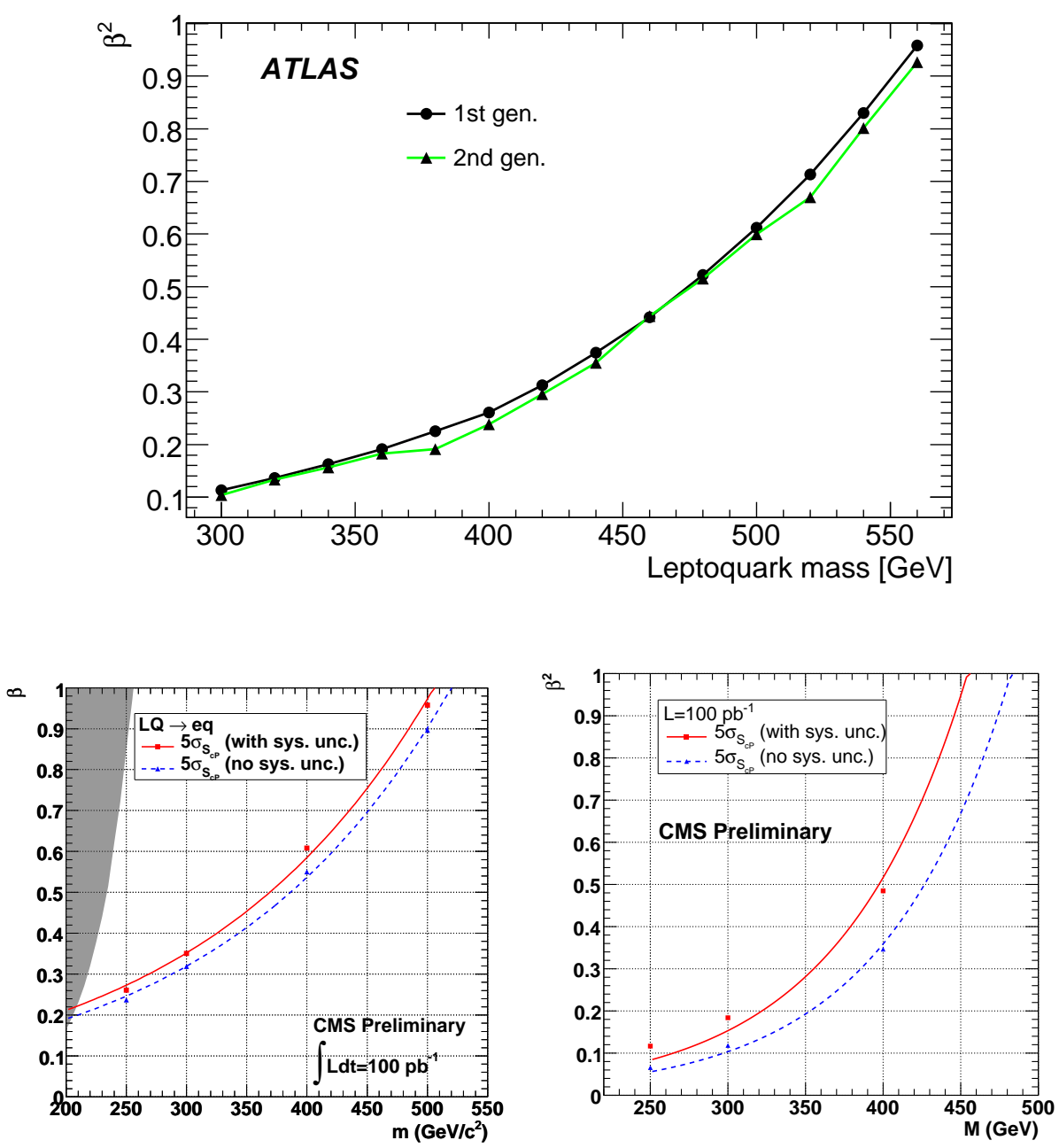

Figure 1: $5 \sigma$ discovery potential for first and second generation leptoquarks at the ATLAS (top) and CMS (bottom) experiments assuming an integrated luminosity of $100 \mathrm{pb}^{-1}$. The bottom left plot shows results for the first generation leptoquark while the bottom right plot presents these results for the second generation. The discovery reach is shown as a function of the mass and branching fraction into the lepton final state, $\beta$.

analysis proceeds analogous to the heavy $b^{\prime}$ analysis described above, leading to an expected $95 \%$ exclusion limit of $400 \mathrm{GeV} / C^{2}$ at $5 \mathrm{TeV}$ beam energy using an integrated luminosity of $100 \mathrm{pb}^{-1}$.

\section{Conclusion}

Various models of new physics predict new particles with sizable branching fractions into fully hadronic or semi-leptonic final states. The ATLAS and CMS experiments at the LHC with their general-purpose design and large acceptance are well suited to search for these new particles. It is expected that even at a reduced beam energy of $5 \mathrm{TeV}$ exclusion limits for such particles can be pushed beyond the ones reached at the Tevatron even at modest integrated luminosities. 


\section{References}

[1] ATLAS Collaboration, The ATLAS experiment at the CERN LHC, JINST 3:S08003 (2008).

[2] CMS Collaboration, The CMS experiment at the CERN LHC, JINST 3:S08004 (2008).

[3] G.G. Ross, Grand Unified Theories, Cambridge U.P, Cambridge, 1987, and references therein.

[4] U. Baur, M. Spira and P. M. Zerwas, Excited Quark and Lepton Production at Hadron Colliders, Phys. Rev. D 42 (1990) 815.

[5] CMS Collaboration, CMS Search Plans and Sensitivity to New Physics Using Dijets, CMS PAS SBM-07-001

[6] D. E. Acosta and S. K. Blessing, Leptoquark searches at HERA and the Tevatron, Ann. Rev. Nucl. Part. Sci. 49 (1999) 389.

[7] S. Davidson, D. C. Bailey and B. A. Campbell, Model independent constraints on leptoquarks from rare processes, Z. Phys. C 61 (1994) 613[hep-ph/9309310].

[8] ATLAS Collaboration, Search for Leptoquark Pairs and Majorana Neutrinos from Right-Handed W Boson Decays in Dilepton-Jets Final States, ATLAS CSC book CERN-OPEN-2008-020

[9] CMS Collaboration, Search for Pair Production of First Generation Scalar Leptoquarks at the CMS Experiment, CMS PAS EXO-08-010

[10] CMS Collaboration, Search for Second Generation Scalar Leptoquarks with the CMS detector, CMS PAS EXO-09-010

[11] CMS Collaboration, Search for Low Mass b' Production in CMS, CMS PAS EXO-08-013

[12] CMS Collaboration, Search for a Fourth Generation b' Quark in tW Final State at CMS in pp Collisions at $\sqrt{s}=10 T e V$, CMS PAS EXO-09-012

[13] R. Contino and G. Servant, Discovering the top partners at the LHC using same-sign dilepton final states, JHEP 0806 (2008) 026 [hep-ph / 0801 . 1679].

[14] CMS Collaboration, Search for Exotic Partners of the Top Quark with the CMS experiment, CMS PAS EXO-08-008 